\title{
A PREDATOR-PREY MODEL OF HOLLING-TYPE II WITH STATE DEPENDENT IMPULSIVE EFFECTS
}

\author{
Changming Ding — ZhongXin Zhang
}

\begin{abstract}
We investigate a predator-prey model with state dependent impulsive effects, which is based on a modified version of the Leslie-Gower scheme and on the Holling-type II scheme. Using topological methods, we present some sufficient conditions to guarantee the existence and asymptotical stability of semi-trivial periodic solutions and positive periodic solutions, respectively.
\end{abstract}

\section{Introduction}

The theory of population dynamic has become an important area of investigation, see [14], [13], for instance. Recently, the systems with state dependent impulsive effects are studied extensively. For example, the existence and asymptotical stability of solutions are established in [7], [11].

In [2], the authors introduce a predator-prey system with two-species food chain, that model describes a prey population which serves as food for a predator. It is written as the following two-dimensional system of differential equations:

$$
\left\{\begin{array}{l}
\frac{d x(t)}{d t}=x(t)\left[r_{1}-b_{1} x(t)-\frac{a_{1} y(t)}{x(t)+k_{1}}\right], \\
\frac{d y(t)}{d t}=y(t)\left[r_{2}-\frac{a_{2} y(t)}{x(t)+k_{2}}\right],
\end{array}\right.
$$

2010 Mathematics Subject Classification. 34A37, 34D05.

Key words and phrases. Impulsive differential equations, state dependent, predator-prey system, periodic solution. 
where $x(t)$ and $y(t)$ represent the population densities at time $t ; b_{1}, r_{i}, a_{i}$ and $k_{i}$ $(i=1,2)$ are model parameters assuming only positive values. It is known that system (1.1) has an unstable node $(0,0)$, two saddle points $\left(r_{1} / b_{1}, 0\right),\left(0, r_{2} k_{2} / a_{2}\right)$ and a locally stable focus $\left(x^{*}, y^{*}\right)$ under the following hypothesis:

(H) $r_{1} \leq r_{2}, k_{1} \geq k_{2}$ and $r_{2} k_{2} / a_{2}<r_{1} k_{1} / a_{1}$, where

$$
\begin{aligned}
x^{*}= & \frac{1}{2 a_{2} b_{1}}\left\{-\left(a_{1} r_{2}-a_{2} r_{1}+a_{2} b_{1} k_{1}\right)\right. \\
& \left.+\left[\left(a_{1} r_{2}-a_{2} r_{1}+a_{2} b_{1} k_{1}\right)^{2}-4 a_{2} b_{1}\left(a_{1} r_{2} k_{2}-a_{2} r_{1} k_{1}\right)\right]^{1 / 2}\right\}, \\
y^{*}= & \frac{r_{2}\left(x^{*}+k_{2}\right)}{a_{2}} .
\end{aligned}
$$

Later, in [11] Nie et al. considered the dynamical behavior of system (1.1) with state dependent impulsive effects, which is modeled by the following equations:

$$
\left\{\begin{aligned}
\frac{d x(t)}{d t} & =x(t)\left[r_{1}-b_{1} x(t)-\frac{a_{1} y(t)}{x(t)+k_{1}}\right] \\
\frac{d y(t)}{d t} & =y(t)\left[r_{2}-\frac{a_{2} y(t)}{x(t)+k_{2}}\right] \\
\triangle x(t) & =x\left(t^{+}\right)-x(t)=-p x(t) \\
\triangle y(t) & =y\left(t^{+}\right)-y(t)=q y(t)+\alpha
\end{aligned}\right\} \quad x \neq h
$$

where $h \in(0, \infty), \alpha \in[0, \infty), p \in(0,1)$ and $q \in(-1, \infty)$. When the amount $x(t)$ of prey reaches the threshold $h$ at time $t_{h}$, a control measure works, then the amounts of prey $x(t)$ and predator $y(t)$ abruptly turn to $(1-p) x\left(t_{h}\right)$ and $(1+q) y\left(t_{h}\right)+\alpha$, respectively. In [11], the authors present several sufficient conditions for the existence and stability of a semi-trivial periodic solution and a positive periodic solution. However, we think that their main results and mathematical proofs are not correct. For example, Theorem 3.4 of [11] states that if $h>x^{*}$, then there are $\alpha>0$ and $q>-1$ such that system (1.2) has an orbitally asymptotically stable positive order- 1 or order- 2 periodic solution. But, if we let $h>r_{1} / b_{1}>x^{*}$, then system (1.2) has no impulsive positive periodic solutions, in fact, by the phase portrait of system (1.1) in the first quadrant (see Figure 1), each positive trajectory with initial condition $\left((1-p) h, y_{0}\right)\left(y_{0} \geq 0\right)$ does not meet the impulsive set $\Sigma^{h}=\left\{(x, y) \mid x=h>r_{1} / b_{1}\right.$ and $\left.y \geq 0\right\}$, or see Theorem 4 of [2]. Thus, system (1.2) has no impulsive periodic solutions. Indeed, even if $h \in\left(x^{*}, r_{1} / b_{1}\right)$, Theorem 3.4 of [11] still may not be true, see Section 4. A similar problem occurs in Theorem 3.1 of [11], where the authors assert that if $\alpha=0$ and

$$
-1<q<\left[\frac{r_{1}-(1-p) b_{1} h}{(1-p)\left(r_{1}-b_{1} h\right)}\right]^{-r_{2} / r_{1}}-1
$$

hold, then system (1.2) has a stable semi-trivial periodic solution. Also, if we let $h>r_{1} / b_{1}$ and choose a positive real number $p$ close to 0 such that $r_{1}-(1-p) b_{1} h<$ 
0 holds, then there exists a $q$ such that (1.3) is true. In this case, system (1.2) with $\alpha=0$ has no semi-trivial periodic trajectories. In fact, since each trajectory of system (1.1) in the positive $x$-axis tends to the saddle point $\left(r_{1} / b_{1}, 0\right)$, the positive trajectory starting from the point $((1-p) h, 0)$ does not reach the impulsive set $\Sigma^{h}$, so the system has no semi-trivial periodic solutions. Hence, Theorem 3.1 of [11] is also not true. Next, in Theorem 3.3 of [11], the uniqueness of a positive periodic solution can not be obtained from its conditions. Finally, in the Section 4 of [11], the authors present examples for the application of their theorems. In those examples, $r_{1}>r_{2}$ holds, it is contradictory to their assumption $(\mathrm{H})$, so their conclusions are unbelievable.

Our goal in this paper is to present suitable conditions to guarantee the existence and asymptotical stability of semi-trivial periodic solutions and positive periodic solutions, respectively. An order is introduced on an arc in the phase space, which is convenient in the study of a Poincaré map associated with our system. Thus, the existence and stability of fixed points of the Poincaré map lead to our results for the periodic solutions of system (1.2). The paper is organized as follows. In the next section, we present some basic definitions and notations. In Section 3, we state and prove new criteria for the existence and asymptotical stability of a semi-trivial periodic solution of system (1.2). The main results of this paper are contained in Section 4, several sufficient conditions for the existence and asymptotical stability of positive periodic solutions of system (1.2) are established. Our conditions are dependent on the model parameters and easy to test. Comparatively, in Theorem 3.3 of [11] is assumed a condition related to the unknown periodic trajectory to get its asymptotical stability. Since one can not locate the site of an unknown periodic trajectory and also does not know its explicit expression, the conclusion of Theorem 3.3 in $[11,(3.7)]$ is hard to use in the applications for concrete models.

\section{Preliminaries}

Clearly, the first quadrant $\Omega=\left\{(x, y) \in \mathbb{R}^{2} \mid x \geq 0, y \geq 0\right\}$ is an invariant region of system (1.1). Hence, for ecological practice, our discussion is limited in the region $\Omega$. Throughout this paper, we assume that the hypothesis $(H)$ holds. Let $\ell_{1}$ be the vertical isocline $y=\left(r_{1}-b_{1} x\right)\left(x+k_{1}\right) / a_{1}$ and $\ell_{2}$ be the horizontal isoline $y=r_{2}\left(x+k_{2}\right) / a_{2}$, respectively. Thus, we obtain the phase portrait of system (1.1) in Figure 1.

It is easy to see that solutions of system (1.1) define a dynamical system $\pi$ in $\Omega$, i.e. a continuous map $\pi$ of $\Omega \times \mathbb{R}$ onto $\Omega$ satisfying $\pi(P, 0)=P$ and $\pi(\pi(P, t), s)=\pi(P, t+s)$ for each $P \in \Omega, t, s \in \mathbb{R}$. For brevity, we write $P \cdot t=\pi(P, t)$, and also let $S \cdot J=\{P \cdot t \mid P \in S, t \in J\}$ for $S \subset \Omega$ and $J \subset \mathbb{R}$. If either $S$ or $J$ is a singleton, i.e. $S=\{P\}$ or $J=\{t\}$, then we simply write 


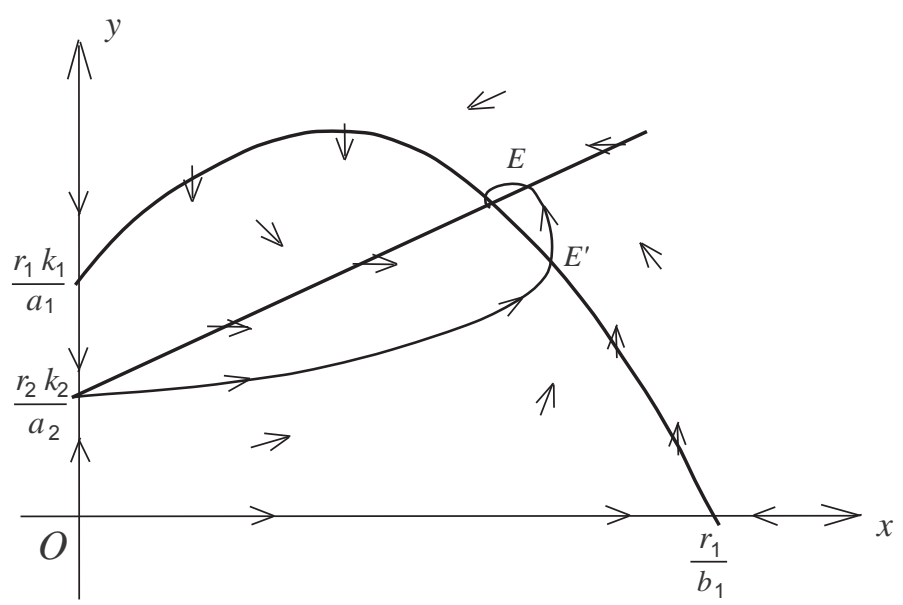

FiguRE 1. The phase portrait of system (1.1).

$P \cdot J$ and $S \cdot t$ for $\{P\} \cdot J$ and $S \cdot\{t\}$, respectively. For each $P \in \Omega$, the map $\pi_{P}: \mathbb{R} \rightarrow \Omega$ defined by $\pi_{P}(t)=\pi(P, t)$ is clearly continuous, and we call $\pi_{P}$ the motion through $P$. The set $P \cdot \mathbb{R}$ is said to be the trajectory of $P$, and $P \cdot \mathbb{R}^{+}$ $\left(P \cdot \mathbb{R}^{-}\right)$is said to be the positive (negative) trajectory of $P$. Replacing $\mathbb{R}$ by $\mathbb{R}^{+}$ in the definition of a dynamical system, we get the definition of a semi-dynamical system. For the elementary properties of dynamical systems and semi-dynamical systems, we refer to [4], [3].

Let $\Sigma^{h}=\left\{(x, y) \in \mathbb{R}^{2} \mid x=h, y \geq 0\right\}$ and $\Sigma^{p}=\left\{(x, y) \in \mathbb{R}^{2} \mid x=\right.$ $(1-p) h, y \geq 0\}$, where $\Sigma^{h}$ is the impulsive set of system (1.2). According to (1.2), we define the impulsive map $I: \Sigma^{h} \rightarrow \Sigma^{p}$ by $I(h, y)=((1-p) h,(1+q) y+\alpha)$ for each $(h, y) \in \Sigma^{h}$. Sometimes, for $P=(h, y) \in \Sigma^{h}$, we denote $I(P)$ by $P^{+}$ and say that $P$ jumps to $P^{+} \in \Sigma^{p}$. For each $P \in \Omega$, by $\Sigma^{h}(P)$ we mean the set $\left(P \cdot \mathbb{R}^{+} \cap \Sigma^{h}\right) \backslash\{P\}$. Obviously, we can define a map $\phi: \Omega \rightarrow \mathbb{R}^{+} \cup\{+\infty\}$ (the space of extended nonnegative reals) by

$$
\phi(P)= \begin{cases}s & \text { if } P \cdot s \in \Sigma^{h} \text { and } P \cdot t \notin \Sigma^{h} \text { for } t \in(0, s), \\ +\infty & \text { if } \Sigma^{h}(P)=\emptyset .\end{cases}
$$

For the continuity of $\phi$, we refer to an important paper of Ciesielski [5]. Let $P \in \Omega$, the motion of system (1.2) through $P$ is an $\Omega$-valued function $\tilde{\pi}_{P}$ defined on $\mathbb{R}^{+}$. From the initial point $P \in \Omega$, the trajectory goes ahead under the role of system (1.1), and when it reaches the impulsive set $\Sigma^{h}$ at a point $P_{1}=P \cdot \phi(P)$, it jumps to $P_{1}^{+}$. Then, it goes again under the role of system (1.1), and also if it meets $\Sigma^{h}$ at a point $P_{2}=P_{1}^{+} \cdot \phi\left(P_{1}^{+}\right)$, it jumps to $P_{2}^{+}$. This process continues inductively, and $\tilde{\pi}_{P}$ is well defined on $\mathbb{R}^{+}$. Note that we define $\tilde{\pi}_{P}(\phi(P))=P_{1}^{+}$, $\tilde{\pi}_{P}\left(\phi(P)+\phi\left(P_{1}^{+}\right)\right)=P_{2}^{+}$, and so on (see [9], [6]). Thus, $\tilde{\pi}_{P}$ is right continuous. 
Obviously, this gives rise to either a finite or infinite number of jumps at points $\left\{P_{n}\right\}$ for the motion $\tilde{\pi}_{P}$. Having each motion $\tilde{\pi}_{P}$ for every point $P$ in $\Omega$, we let $\tilde{\pi}(P, t)=\widetilde{\pi}_{P}(t)$ for $P \in \Omega$ and $t \in \mathbb{R}^{+}$, and then we get a discontinuous system defined by the solutions of system (1.2) with the following properties:

(i) $\tilde{\pi}(P, 0)=P$ for $P \in \Omega$,

(ii) $\quad \tilde{\pi}(\tilde{\pi}(P, t), s)=\widetilde{\pi}(P, t+s)$ for $P \in \Omega$ and $t, s \in \mathbb{R}^{+}$.

We call $\tilde{\pi}(P, t)$, with $\tilde{\pi}$ as defined above, an impulsive semi-dynamical system associated with system (1.2). Also, we denote $P * t=\tilde{\pi}(P, t)$ for brevity. Then, (ii) reads $(P * t) * s=P *(t+s)$. The set $P * \mathbb{R}^{+}$is said to be the trajectory of $P$. Note that for a $P \in \Omega, P \cdot \phi(P)=P_{1}$ lies in $\Sigma^{h}$, but $P * \phi(P)=P_{1}^{+}$lies in $\Sigma^{p}$. So, our system $\tilde{\pi}$ is right continuous for each $P \in \Omega$, but not left continuous. For the theory of impulsive semi-dynamical systems, we refer to [9], [10], [1], [8].

Throughout the paper, for a point $P$ in $\Omega$, let $B(P, \delta)=\{Q \in \Omega \mid d(P, Q)<$ $\delta$ \} be the open disk in $\Omega$ with center $P$ and radius $\delta>0$, where $d$ is the ordinary metric on $\mathbb{R}^{2}$. In addition, for $S \subset \Omega$, the $r$-neighborhood of $S$ in $\Omega$ is denoted by $N(S, r)=\{P \in \Omega \mid d(P, S)<r\}$ for $r>0$, where $d(P, S)=\inf \{d(P, Q) \mid Q \in$ $S\}$. Here, with no confusion, we also use $d$ for the distance between a point and a set. Now, we recall several definitions that will be used in the sequel.

Definition 2.1 ([12]). Let $P_{0} \in \Omega \backslash \Sigma^{h}$. The trajectory $P_{0} * \mathbb{R}^{+}$of system (1.2) is said to be orbitally stable if, given an $\varepsilon>0$, there exists a $\delta=\delta(\varepsilon)>0$ such that for any $P \in B\left(P_{0}, \delta\right)$, then we have $P * \mathbb{R}^{+} \subset N\left(P_{0} * \mathbb{R}^{+}, \varepsilon\right)$.

Definition $2.2([12])$. Let $P_{0} \in \Omega \backslash \Sigma^{h}$. The trajectory $P_{0} * \mathbb{R}^{+}$of system (1.2) is said to be asymptotically orbitally stable if it is orbitally stable and there exists an $\eta>0$ such that if $P \in B\left(P_{0}, \eta\right)$, then $\lim _{t \rightarrow+\infty} d\left(P * t, P_{0} * \mathbb{R}^{+}\right)=0$.

Definition $2.3([9])$. A trajectory $P_{0} * \mathbb{R}^{+}$for $P_{0} \in \Omega$ is periodic if there exists a $t>0$ such that $P_{0}=P_{0} * t$, and $\tilde{\pi}_{P_{0}}$ is called a periodic motion with period $t$. In particular, if a periodic trajectory $P_{0} * \mathbb{R}^{+}$lies in the boundary $\partial \Omega$ of $\Omega$, i.e. $P_{0} * \mathbb{R}^{+}$lies in the positive $x$-axis or $y$-axis, then we call it a semi-trivial periodic trajectory.

For a periodic trajectory, the concept of order has been defined in [9]. However, in this paper we do not deal with the order of periodic trajectories in detail, so we just call it a periodic trajectory with period $t$. Generally, an impulsive periodic trajectory is of order $n$ if it has $n$ discontinuous points. Here, we recall the notion of order given in [11], which is a special case of the corresponding definition in [9]. Let $P_{0}=\left((1-p) h, y_{0}\right) \in \Sigma^{p}$. Its trajectory $P_{0} * \mathbb{R}^{+}$first reaches $\Sigma^{h}$ at a point $Q_{0}$, next jumps to $P_{1}=Q_{0}^{+}=\left((1-p) h, y_{1}\right) \in \Sigma^{p}$. Again, the trajectory goes ahead and meets $\Sigma^{h}$ at $Q_{1}$, then jumps to $P_{2}=Q_{1}^{+}=\left((1-p) h, y_{2}\right) \in \Sigma^{p}$. This process continues inductively, and we obtain a sequence $\left\{\left((1-p) h, y_{n}\right) \mid\right.$ 
$n=0,1, \ldots\}$. If there exists a positive integer $k$ such that $k$ is the smallest integer for $y_{0}=y_{k}$, then the trajectory $P_{0} * \mathbb{R}^{+}$is said to be order- $k$ periodic.

\section{Semi-trivial periodic trajectories}

In this section, we consider the existence and asymptotical stability of semitrivial periodic trajectories. By definition, the existence of a semi-trivial periodic trajectory for system (1.2) implies $\alpha=0$, otherwise, at the point $(h, 0)$ the trajectory jumps to a point in the interior of $\Omega$. Thus, in this section we always assume $\alpha=0$.

Clearly, in the positive $x$-axis, we have the sub-system

$$
\begin{cases}\frac{d x(t)}{d t}=x(t)\left[r_{1}-b_{1} x(t)\right], & x \neq h, \\ \triangle x=x\left(t^{+}\right)-x(t)=-p x(t), & x=h .\end{cases}
$$

Observe that there exists an equilibrium in the positive $x$-axis. Without the impulsive effect, each trajectory in the positive $x$-axis tends to the equilibrium $\left(r_{1} / b_{1}, 0\right)$. Thus, if $h \geq r_{1} / b_{1}$, the trajectory starting from $((1-p) h, 0)$ does not reach the point $(h, 0)$, and it follows that there exist no semi-trivial periodic trajectories.

Theorem 3.1. If $\alpha=0,0<h<\frac{r_{1}}{b_{1}}$ and

$$
-1<q<\left[\frac{r_{1}-(1-p) b_{1} h}{(1-p)\left(r_{1}-b_{1} h\right)}\right]^{-r_{2} / r_{1}}-1 \triangleq \lambda-1,
$$

then system (1.2) has an asymptotically orbitally stable semi-trivial periodic trajectory.

Proof. Let $A=((1-p) h, 0)$. Obviously, $\widetilde{\pi}_{A}\left(\mathbb{R}^{+}\right)=A * \mathbb{R}^{+}=[(1-p) h, h)$ is a semi-trivial periodic trajectory. Choose a point $Q=\left((1-p) h, n_{0}\right) \in \Sigma^{p}$ close to $A$, where $n_{0}>0$ is sufficiently small. Assume that $\pi_{Q}$ intersects $\Sigma^{h}$ at $Q_{1}=Q \cdot \phi(Q)=(h, n)$. By the continuous dependence on the initial conditions, $n=n\left(n_{0}\right)$ is also small. Thus, by a simple estimate of the integral

$$
\int_{(1-p) h}^{h} \frac{r_{2}-a_{2} y /\left(x+k_{2}\right)}{x\left(r_{1}-b_{1} x-a_{1} y /\left(x+k_{1}\right)\right)} d x,
$$

where $y=y\left(x, n_{0}\right)$ is small and $\{(x, y(x)) \mid(1-p) h \leq x \leq h\}$ is the trajectory segment of system (1.1) through $Q$, we have

$$
n=n_{0}\left[\frac{r_{1}-(1-p) b_{1} h}{(1-p)\left(r_{1}-b_{1} h\right)}\right]^{r_{2} / r_{1}}+o\left(n_{0}\right)=\lambda^{-1} n_{0}+o\left(n_{0}\right) .
$$

This result also can be obtained immediately by formula (2.13) in [15, p. 29].

Now, let $Q_{1}^{+}=\left((1-p) h, n_{1}\right)$, where $n_{1}=(1+q) n$. Hence, if $1+q<\lambda$, it follows that $n_{1}<n_{0}$ holds for any small $n_{0}$, i.e. $Q_{1}^{+}$lies below $Q$ in $\Sigma^{p}$. Then, the trajectory $\tilde{\pi}_{Q}$ goes ahead and meets $\Sigma^{h}$ at a point $Q_{2}$ below $Q_{1}$. 
Again, $Q_{2}$ jumps to a point $Q_{2}^{+}$in $\Sigma^{p}$, where $Q_{2}^{+}$lies below $Q_{1}^{+}$. By induction, the sequence $\left\{Q_{k}^{+}\right\}$goes down in $\Sigma^{p}$ monotonously as $k \rightarrow+\infty$. If $Q_{k}^{+}$tends to $A$ as $k \rightarrow+\infty$, by the continuous dependence on the initial conditions and Definition 2.2 , it is easy to see that $A * \mathbb{R}^{+}$is asymptotically orbitally stable, and the proof is finished. Otherwise, if $Q_{k}^{+} \rightarrow P \in \Sigma^{p}$, it follows from [9, Theorem 2] that $\widetilde{\pi}_{P}$ is a periodic trajectory. However, by the argument above, we conclude that $P * \phi(P)$ lies below $P$ in $\Sigma^{p}$, which means that $\widetilde{\pi}_{P}$ is not a periodic trajectory. This is a contradiction, and thus we complete the proof.

\section{Positive periodic trajectories}

In this section, we consider the existence and asymptotical stability of positive impulsive periodic trajectories of system (1.2). Let $W^{u}\left(0, r_{2} k_{2} / a_{2}\right)$ be the unstable manifold of the saddle point $\left(0, r_{2} k_{2} / a_{2}\right)$ in $\Omega$. Note that $W^{u}\left(0, r_{2} k_{2} / a_{2}\right)$ plays an important role in the qualitative analysis of system (1.1). Let $E^{\prime}=(\bar{x}, \bar{y})$ be the first point of intersection of $W^{u}\left(0, r_{2} k_{2} / a_{2}\right)$ and $\ell_{1}$, see Figure 1 . Recall that if $h \geq r_{1} / b_{1}$, then system (1.2) has no impulsive periodic trajectories. So, in the following, we deal with the cases $0<h \leq x^{*}, x^{*}<h<\bar{x}$ and $\bar{x} \leq h<r_{1} / b_{1}$, respectively.

4.1. The case $0<h \leq x^{*}$. Let $A=((1-p) h, 0)$. Let $B=\left((1-p) h, y_{b}\right)$ be the point of intersection of $\Sigma^{p}$ and $\ell_{1}$, where $y_{b}=\left[r_{1}-(1-p) b_{1} h\right]\left[(1-p) h+k_{1}\right] / a_{1}$. The first point of intersection of the unstable manifold $W^{u}\left(0, r_{2} k_{2} / a_{2}\right)$ and $\Sigma^{p}$ is denoted by $U=\left((1-p) h, y_{u}\right)$, see Figure 2. Clearly, we have $y_{b}>y_{u}>0$. Let $J$ be the segment $\overline{A B}=\left\{((1-p) h, y) \in \Sigma^{p} \mid 0 \leq y \leq y_{b}\right\}$. It is easy to

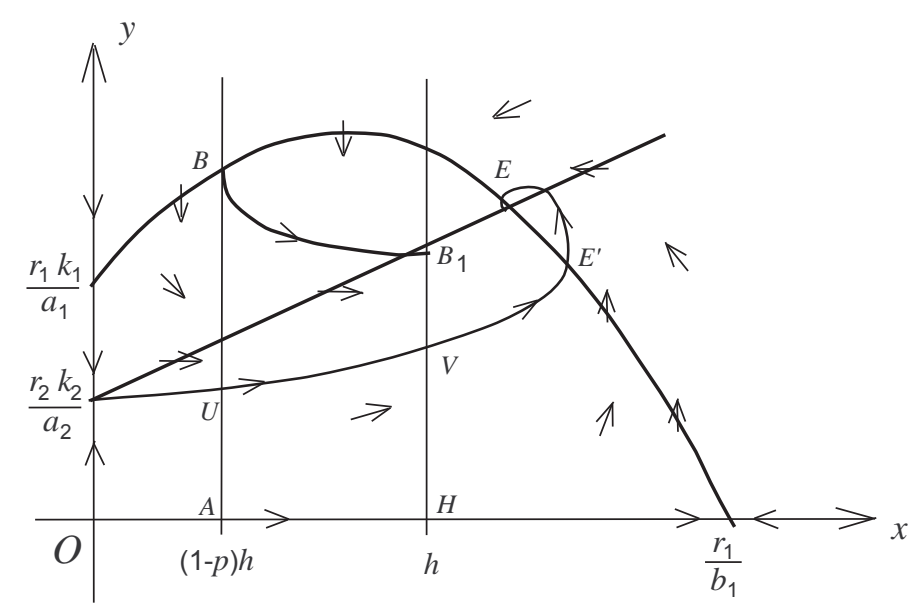

Figure 2. The case $0<h \leq x^{*}$.

see that each trajectory of a point in $J$ meets $\Sigma^{h}$. Let $B_{1}=B \cdot \phi(B)=\left(h, y_{h}\right)$ 
and $H=(h, 0)$. Now, we define a Poincaré map $F: J \rightarrow J$ as follows. For each $P \in J$, let the trajectory $P \cdot \mathbb{R}^{+}$intersect $\Sigma^{h}$ at the point $P_{1}=P \cdot \phi(P)=\left(h, y_{1}\right)$. Then, if $P_{1}^{+}=I\left(P_{1}\right) \in J$, we put $F(P)=P_{1}^{+}$; If $P_{1}^{+} \in \Sigma^{p} \backslash J$, by the phase portrait of system $(1.1), P_{1}^{+} \cdot \mathbb{R}^{+}$first intersects $J$ at a point $P_{1}^{\prime}$, i.e. $P_{1}^{\prime}=P_{1}^{+} \cdot t$ for a $t>0$ and $P_{1}^{+} \cdot(0, t) \cap \Sigma^{p}=\emptyset$, thus we define $F(P)=P_{1}^{\prime}$. By the continuity of impulsive map $I$ and the continuous dependence on the initial conditions, it is easy to verify that $F$ is a continuous map. Since $J$ is homeomorphic to the interval $[0,1]$ that has the fixed point property, it follows that there exists a fixed point $P_{0} \in J$ of $F$. Clearly, $P_{0} * \mathbb{R}^{+}$is an impulsive periodic trajectory. Hence, we have the following result.

THEOREM 4.1. If $h \leq x^{*}$, then for any $q>-1$ and $\alpha>0$ system (1.2) has a positive periodic trajectory.

In the following, we present sufficient conditions for an asymptotically stable positive periodic trajectory. Let $V=U \cdot \phi(U)=\left(h, y_{v}\right)$, then $y_{h}>y_{v}>0$ holds.

TheOREM 4.2. Let $h \leq x^{*}$. If $(1+q) y_{v}+\alpha \geq y_{b}$ holds, then system (1.2) has an orbitally stable periodic trajectory.

Proof. Observe that $I(V)\left(\in \Sigma^{P}\right)$ lies above $B$, since $(1+q) y_{v}+\alpha \geq y_{b}$. Let $J_{0}=\overline{U B}$ be a sub-segment in $J$ and $F_{0}=\left.F\right|_{J_{0}}$ be the restriction of $F$ in $J_{0}$. We define an order ' $\prec$ ' on $J_{0}$ as follows. Let $P=\left((1-p) h, y_{1}\right)$ and $Q=\left((1-p) h, y_{2}\right)$ be two different points in $J_{0}$, then we define $P \prec Q$ if and only if $y_{1}<y_{2}$. Clearly, for any $P$ and $Q$ in $J_{0}$, it follows from the phase portrait of system (1.1) that if $Q \prec P$, then $F_{0}(P) \prec F_{0}(Q)$ holds, i.e. $F_{0}$ is a reversal map. Thus, it implies that there exists a unique fixed point $P_{0}$ of $F_{0}$ in $J_{0}$. Otherwise, if $F_{0}$ has two different fixed points $P_{1}$ and $P_{2}$ in $J_{0}$ with $P_{1} \prec P_{2}$, then we have $P_{2}=F_{0}\left(P_{2}\right) \prec F_{0}\left(P_{1}\right)=P_{1}$, which is a contradiction. Note that for any $P \prec Q$ in $J_{0}, F_{0}^{2}(P) \prec F_{0}^{2}(Q)$ holds, i.e. $F_{0}^{2}$ is strictly monotonous on $J_{0}$. Clearly, we have $U \prec F_{0}^{2}(U)$, which also implies $F_{0}^{2}(U) \prec F_{0}^{4}(U)$. It follows that $U \prec F_{0}^{2}(U) \prec F_{0}^{4}(U) \prec \ldots \prec B$. Thus, there exists a $Q_{0} \in J_{0}$ such that $F_{0}^{2 n}(U) \rightarrow Q_{0}$ as $n \rightarrow+\infty$. It is easy to see that $F_{0}^{2}\left(Q_{0}\right)=Q_{0}$, which means that $Q_{0} * \mathbb{R}^{+}$is an impulsive periodic trajectory. If $Q_{0} \neq P_{0}$, then $Q_{0} * \mathbb{R}^{+}$is of order 2. Now, if for any $Q \in J_{0}$ with $Q_{0} \prec Q$ there exists a $Q^{\prime}$ in the interval $\left(Q_{0}, Q\right) \triangleq\left\{P \in J_{0} \mid Q_{0} \prec P \prec Q\right\}$ such that $F_{0}^{2}\left(Q^{\prime}\right) \prec Q^{\prime}$ or $F_{0}^{2}\left(Q^{\prime}\right)=Q^{\prime}$ holds, then we assert that $Q_{0} * \mathbb{R}^{+}$is orbitally stable. Actually, it means that $Q_{0}$ is a stable fixed point of $F_{0}^{2}$, i.e. there is an arbitrary small interval $J^{\prime}=\left(Q^{\prime \prime}, Q^{\prime}\right)$ containing $Q_{0}$ such that $F_{0}^{2}\left(J^{\prime}\right) \subset J^{\prime}$. Hence, by the continuous dependence on the initial conditions and Definition 2.1, it is easy to conclude that $Q_{0} * \mathbb{R}^{+}$is orbitally stable, which is the desired conclusion. Next, if $Q^{\prime} \prec F_{0}^{2}\left(Q^{\prime}\right)$ holds for a $Q^{\prime}$ in an interval $\left(Q_{0}, Q\right) \subset J_{0}$, then we fix such a point $U_{1}$ with $Q_{0} \prec$ $U_{1} \prec F_{0}^{2}\left(U_{1}\right)$. Similarly, we obtain that $U_{1} \prec F_{0}^{2}\left(U_{1}\right) \prec F_{0}^{4}\left(U_{1}\right) \prec \ldots \prec B$ and 
$F_{0}^{2 n}\left(U_{1}\right) \rightarrow Q_{1}$ as $n \rightarrow+\infty$. Thus, by a similar argument as above, either $Q_{1} * \mathbb{R}^{+}$ is orbitally stable or there exists a $U_{2}$ satisfying $Q_{1} \prec U_{2} \prec F_{0}^{2}\left(U_{2}\right)$. The process continues inductively, we conclude that either there exists an orbitally stable periodic trajectory or there exists a sequence $\left\{U_{i}\right\}$ such that $U_{1} \prec U_{2} \prec \ldots \prec B$, $U_{i} \prec F_{0}^{2}\left(U_{i}\right)$ for each $i \geq 1$ and $U_{i} \rightarrow B$ as $i \rightarrow+\infty$. For the latter case, since $U_{i} \prec F_{0}^{2}\left(U_{i}\right) \prec B$ for each $i \geq 1$, it leads to $F_{0}^{2}(B)=B$, but it is impossible. In fact, by the phase portrait of system (1.1), we have $U \prec F_{0}(B) \prec B$, and it implies $F_{0}(B) \prec F_{0}^{2}(B) \prec B$. So, it is a contradiction. The proof is complete.

Theorem 4.3. Let $h \leq x^{*}$. If $(1+q) y_{h}+\alpha \leq y_{b}, r_{1}+r_{2} \leq 2 b_{1}(1-p) h$ and

$$
\frac{(1+q)(1-p)\left[r_{1}-b_{1}(1-p) h\right]}{\left(r_{1}-b_{1} h-a_{1} y_{h} /\left(h+k_{1}\right)\right)}<1
$$

hold, then system (1.2) has an asymptotically orbitally stable periodic trajectory, which is a unique impulsive periodic trajectory.

Proof. Since $(1+q) y_{h}+\alpha \leq y_{b}$ holds, the segment $\overline{H B_{1}}$ is mapped into $J$ by $I$, i.e. $I\left(\overline{H B_{1}}\right) \subset J$. Hence, for $P \in J$, we have $F(P)=I(P \cdot \phi(P))$. It follows that the polygon $S=A B B_{1} H$ is positively invariant under $\widetilde{\pi}$, i.e. $S * t \subset S$ for $t \geq 0$. By Theorem 4.1, system (1.2) has an impulsive periodic trajectory $P_{0} * \mathbb{R}^{+}$ lying in $S$, where $P_{0} \in J$. Now, for any $P_{1}=\left((1-p) h, y_{1}\right), P_{2}=\left((1-p) h, y_{2}\right) \in$ $J$, it is easy to see that trajectory segments $P_{1} \cdot\left[0, \phi\left(P_{1}\right)\right], P_{2} \cdot\left[0, \phi\left(P_{2}\right)\right]$, and $\Sigma^{p}, \Sigma^{h}$ surround a region $\Re$, which lies in $S$. Let $P_{1}^{\prime}=P_{1} \cdot \phi\left(P_{1}\right)=\left(h, y_{1}^{\prime}\right)$ and $P_{2}^{\prime}=P_{2} \cdot \phi\left(P_{2}\right)=\left(h, y_{2}^{\prime}\right)$, respectively. Without loss of generality, we suppose $y_{1}>y_{2}$, and it also implies $y_{1}^{\prime}>y_{2}^{\prime}$. We apply Green's Formula to $\Re$, and then we have

$$
\oint_{\partial \Re} f_{1} d y-f_{2} d x=\iint_{\Re}\left(\frac{\partial f_{1}}{\partial x}+\frac{\partial f_{2}}{\partial y}\right) d x d y,
$$

where $\partial \Re$ is the boundary of $\Re, f_{1}(x, y)=x\left[r_{1}-b_{1} x-a_{1} y /\left(x+k_{1}\right)\right]$ and $f_{2}(x, y)=y\left[r_{2}-a_{2} y /\left(x+k_{2}\right)\right]$. Since $r_{1}+r_{2} \leq 2 b_{1}(1-p) h$ holds, it is easy to verify that

$$
\frac{\partial f_{1}}{\partial x}+\frac{\partial f_{2}}{\partial y} \leq 0 \quad \text { for any }(x, y) \in \Re \text {. }
$$

It follows that

$$
\oint_{\partial \Re} f_{1} d y-f_{2} d x \leq 0
$$

Clearly, on each trajectory segment we have $f_{1} d y-f_{2} d x=0$, thus it follows

$$
\left(\int_{P_{1} P_{2}}+\int_{P_{2}^{\prime} P_{1}^{\prime}}\right) f_{1} d y-f_{2} d x \leq 0 \quad \text { or } \quad \int_{y_{1}}^{y_{2}} f_{1} d y \leq \int_{y_{1}^{\prime}}^{y_{2}^{\prime}} f_{1} d y .
$$


By a simple computation, we obtain

$$
\begin{aligned}
(1-p)\left(y_{1}-y_{2}\right)\left[r_{1}-b_{1}(1-p) h\right. & \left.-\frac{a_{1}\left(y_{1}+y_{2}\right)}{2\left[(1-p) h+k_{1}\right]}\right] \\
& \geq\left(y_{1}^{\prime}-y_{2}^{\prime}\right)\left[r_{1}-b_{1} h-\frac{a_{1}\left(y_{1}^{\prime}+y_{2}^{\prime}\right)}{2\left(h+k_{1}\right)}\right] .
\end{aligned}
$$

Since $y_{2}^{\prime}<y_{1}^{\prime} \leq y_{h}$, by (4.2) we have

$$
(1-p)\left(y_{1}-y_{2}\right)\left[r_{1}-b_{1}(1-p) h\right] \geq\left(y_{1}^{\prime}-y_{2}^{\prime}\right)\left[r_{1}-b_{1} h-\frac{a_{1} y_{h}}{h+k_{1}}\right] .
$$

Thus, it follows from (4.1) that $(1+q)\left(y_{1}^{\prime}-y_{2}^{\prime}\right)<y_{1}-y_{2}$, i.e. $d\left(F\left(P_{1}\right), F\left(P_{2}\right)\right)<$ $d\left(P_{1}, P_{2}\right)$. Hence, $F$ is a contraction on $J$, and by the Banach Fixed Point Theorem it has a unique fixed point $P_{0}$, which is also asymptotically stable for $F$. So, by the continuous dependence on the initial conditions and Definition 2.2, it is easy to see that $P_{0} * \mathbb{R}^{+}$is asymptotically orbitally stable for $\widetilde{\pi}$. This completes the proof.

REMARK 4.4. Note that $y_{b}$ is determined by model parameters, but $y_{v}$ and $y_{h}$ are not. Fortunately, they can be determined by the unstable manifold of saddle point $\left(0, r_{2} k_{2} / a_{2}\right)$ and the trajectory $B * \mathbb{R}^{+}$, respectively. For concrete systems, we may estimate them even by computers.

4.2. The case $x^{*}<h<\bar{x}$. Now, we consider two cases $B \cdot \mathbb{R}^{+} \cap \Sigma^{h}=\emptyset$ and $B \cdot \mathbb{R}^{+} \cap \Sigma^{h} \neq \emptyset$, respectively. First, for the case $B \cdot \mathbb{R}^{+} \cap \Sigma^{h} \neq \emptyset$, it is easy to see that Theorems 4.2 and 4.3 work well. So, we omit to restating those two theorems for this case. In the sequel, we focus on the case $B \cdot \mathbb{R}^{+} \cap \Sigma^{h}=\emptyset$. Let $W=\left(h, y_{w}\right)$ be the intersection point of $\Sigma^{h}$ and $\ell_{1}$, where $y_{w}=\left(r_{1}-b_{1} h\right)\left(h+k_{1}\right) / a_{1}$. Then, by the phase portrait of system (1.1), the negative trajectory $W \cdot \mathbb{R}^{-}$first intersects $\Sigma^{p}$ at a point $W_{p}$ between $U$ and $B$, next it meets $\Sigma^{p}$ at a point $Z$ above $B$, see Figure 3 .

Let $W_{p}=\left((1-p) h, y_{p}\right)$ and $Z=\left((1-p) h, y_{z}\right)$, where $y_{z}>y_{b}>y_{p}>y_{u}$. First, assume $(1+q) y_{v}+\alpha>y_{z}$, then the restriction $F_{1}=\left.F\right|_{\overline{U W_{p}}}$ of $F$ on the segment $\overline{U W_{p}}$ is a reversal map. Thus, after the similar reasoning as in the proof of Theorem 4.2, we obtain the following result.

Theorem 4.5. Assume that $x^{*}<h<\bar{x}$ and $B \cdot \mathbb{R}^{+} \cap \Sigma^{h}=\emptyset$. If $(1+q) y_{v}+\alpha$ $>y_{z}$ holds, then system (1.2) has an orbitally stable periodic trajectory.

Secondly, if $(1+q) y_{w}+\alpha \leq y_{p}$, we consider the restriction $\left.F\right|_{\overline{A W_{p}}}$ of $F$ on the segment $\overline{A W_{p}}$. Also, using the similar reasoning as in the proof of Theorem 4.3, we obtain the following conclusion. 


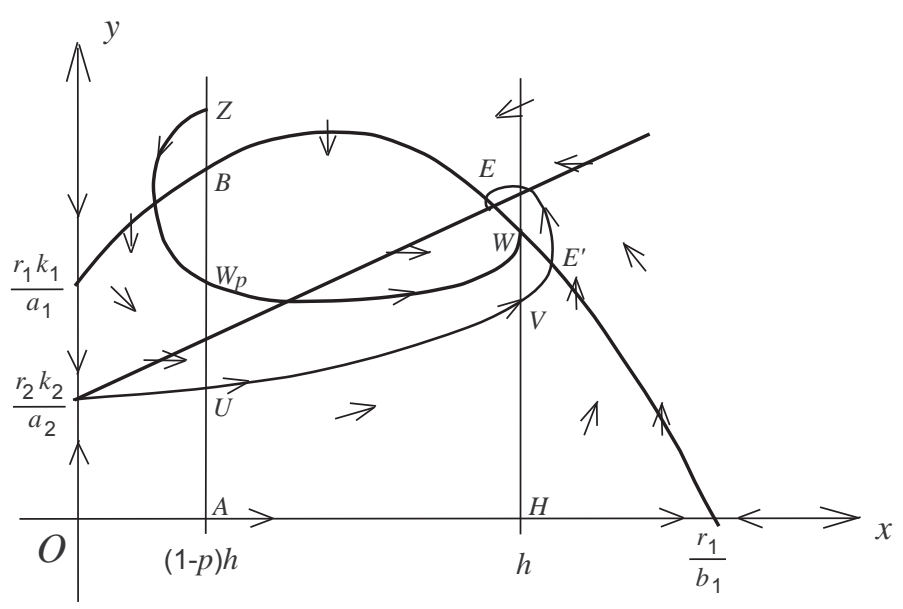

Figure 3. The case $x^{*}<h<\bar{x}$.

Theorem 4.6. Assume that $x^{*}<h<\bar{x}$ and $B \cdot \mathbb{R}^{+} \cap \Sigma^{h}=\emptyset$. If $(1+q) y_{w}+$ $\alpha \leq y_{p}, r_{1}+r_{2} \leq 2 b_{1}(1-p) h$ and

$$
\frac{(1+q)(1-p)\left[r_{1}-b_{1}(1-p) h\right]}{\left(r_{1}-b_{1} h-a_{1} y_{w} /\left(h+k_{1}\right)\right)}<1
$$

hold, then system (1.2) has an asymptotically orbitally stable periodic trajectory, which is a unique impulsive periodic trajectory.

4.3. The case $\bar{x} \leq h<r_{1} / b_{1}$. Let $W=\left(h, y_{w}\right)$ be the intersection point of $\Sigma^{h}$ and $\ell_{1}$, where $y_{w}=\left[r_{1}-b_{1} h\right]\left(h+k_{1}\right) / a_{1}$. Then, the negative trajectory $W \cdot \mathbb{R}^{-}$intersects $\Sigma^{p}$ at a point $W_{p}=\left((1-p) h, y_{p}\right)$, see Figure 4. Clearly, if $\alpha \geq y_{p}$, then there exist no impulsive periodic trajectories. Indeed, we have a stronger result as follows.

TheOREM 4.7. Let $\bar{x} \leq h<r_{1} / b_{1}$. If $q \geq 0$, system (1.2) has no impulsive periodic trajectories. If $q \in(-1,0)$ and $\alpha>-q y_{p}$, system (1.2) has no impulsive periodic trajectories.

Proof. Let $A=((1-p) h, 0)$ and $H=(h, 0)$. Denote $\mu=1+q>0$. We consider the trajectory $A * \mathbb{R}^{+}$. First, let $A_{1}=I(H)=H^{+}=\left((1-p) h, y_{1}\right)$, then $y_{1}=\alpha$. If $A_{1}$ is below $W_{p}$ in $\Sigma^{p}$, then let $A_{1}^{\prime}=A_{1} \cdot \phi\left(A_{1}\right)=\left(h, y_{1}^{\prime}\right) \in \Sigma^{h}$. By the phase portrait of system (1.1), we have $y_{1}^{\prime}>y_{1}$. Next, let $A_{2}=I\left(A_{1}^{\prime}\right)=$ $\left((1-p) h, y_{2}\right)$, then $y_{2}=\mu y_{1}^{\prime}+\alpha>(\mu+1) \alpha$. Again, if $A_{2}$ is below $W_{p}$ in $\Sigma^{p}$, then let $A_{2}^{\prime}=A_{2} \cdot \phi\left(A_{2}\right)=\left(h, y_{2}^{\prime}\right) \in \Sigma^{h}$. Similarly, we have $y_{2}^{\prime}>y_{2}$. Thus, let $A_{3}=I\left(A_{2}^{\prime}\right)=\left((1-p) h, y_{3}\right)$, and then $y_{3}>\left(\mu^{2}+\mu+1\right) \alpha$. Inductively, we obtain that either an $A_{n_{0}}$ lies above $W_{p}$ in $\Sigma^{p}$ for some positive integer $n_{0}$ or there exists a sequence $\left\{A_{n}\right\}$ lying below $W_{p}$ in $\Sigma^{p}$, where $A_{n}=\left((1-p) h, y_{n}\right)$ and $y_{n}>\left(\mu^{n}+\mu^{n-1}+\ldots+1\right) \alpha$. For the latter case, if $q \geq 0$, then $\left(\mu^{n}+\right.$ 


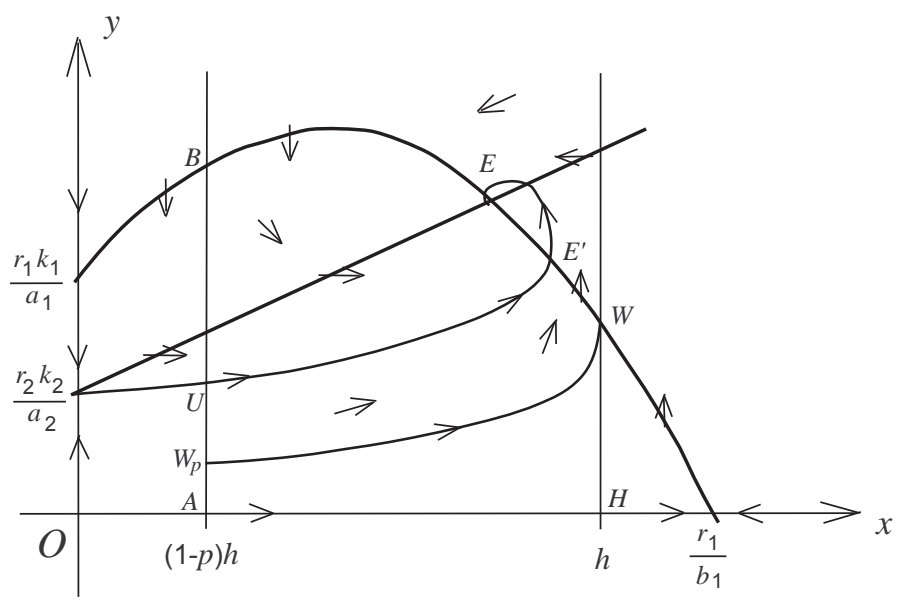

Figure 4. The case $\bar{x} \leq h<r_{1} / b_{1}$.

$\left.\mu^{n-1}+\ldots+1\right) \alpha \rightarrow+\infty$ as $n \rightarrow+\infty$, it implies that some $A_{n}$ lies above $W_{p}$ in $\Sigma^{p}$; If $q \in(-1,0)$ and $\alpha>-q y_{p}$ hold, then $\left(\mu^{n}+\mu^{n-1}+\ldots+1\right) \alpha \rightarrow-\alpha / q$ as $n \rightarrow+\infty$ and it means $y_{n}>y_{p}$ for some $n$, i.e. some $A_{n}$ lies above $W_{p}$. Anyway, we conclude that the trajectory $A * \mathbb{R}^{+}$has at most a finite number of impulsive points and $A *\left[t_{0},+\infty\right) \cap \Sigma^{h}=\emptyset$ for some $t_{0}>0$. Clearly, each trajectory with an initial point in the segment $\overline{A W_{p}}$ has a similar dynamical behavior, i.e. it has at most finite number of impulsive points and finally does not meet with $\Sigma^{h}$. This completes the proof.

Finally, we assume that $(1+q) y_{w}+\alpha \leq y_{p}$. Consider the restriction $\left.F\right|_{\overline{A W_{p}}}$ of $F$ on the segment $\overline{A W_{p}}$. After the similar reasoning as in the proof of Theorem 4.3 we obtain the following result.

TheOrem 4.8. Assume that $\bar{x} \leq h<r_{1} / b_{1}$ and $(1+q) y_{w}+\alpha \leq y_{p}$. If $r_{1}+r_{2} \leq 2 b_{1}(1-p) h$ and

$$
\frac{(1+q)(1-p)\left[r_{1}-b_{1}(1-p) h\right]}{\left(r_{1}-b_{1} h-a_{1} y_{w} /\left(h+k_{1}\right)\right)}<1
$$

hold, then system (1.2) has an asymptotically orbitally stable periodic trajectory, which is a unique impulsive periodic trajectory.

Acknowledgements. The authors sincerely thank the referees for their very careful reading of the original manuscript and for many syntactical and semantic suggestions. Also, the authors thank Professor Jiangang Qi for a helpful discussions. Finally, the authors greatly appreciate the excellent editorial works. 


\section{REFERENCES}

[1] M. Акнmet, Principles of Discontinuous Dynamical Systems, Springer, New York, 2010.

[2] M.A. Aziz-Alaoui And M. Daher ОкiYe, Boundedness and global stability for a predatorprey model with modified Leslie-Gower and Holling-type II schemes, Appl. Math. Lett. 16 (2003), 1069-1075.

[3] N.P. Bhatia and O. Hajek, Local Semi-Dynamical Systems, Lecture Notes in Mathematics, vol. 90, Springer, Berlin, 1970.

[4] N.P. Bhatia And G.P. Szego, Stability Theory of Dynamical Systems, Springer, Berlin, 2002.

[5] K. Ciesielski, On semi-continuity in impulsive systems, Bull. Polish Acad. Sci. Math. 52 (2004), 71-80.

[6] C. Ding, Lyapunov quasi-stable trajectories, Fund. Math. 220 (2013), 139-154.

[7] _ A predator-prey model with state dependent impulsive effects, Ann. Polon. Math. 111 (2014), 297-308.

[8] W.M. Haddad, V. Chellaboina and S.G. Nersesov, Impulsive and Hybrid Dynamical Systems: Stability, Dissipativity, and Control, Princeton Univ. Press, Princeton, NJ, 2006.

[9] S.K. KAUL, On impulsive semi-dynamical systems, J. Math. Anal. Appl. 150 (1990), $120-128$.

[10] V. Lakshmikantham, D.D. Bainov and P.S. Simeonov, Theory of Impulsive Differential Equations, World Scientific, Singapore, 1989.

[11] L.F. Nie, Z.D. Teng, L. Hu And J.G. Peng, Qualitative analysis of a modified LeslieGower and Holling-type II predator-prey model with state dependent impulsive effects, Nonlinear Anal. 11 (2010), 1364-1373.

[12] P.S. Simeonov and D.D. Bainov, Orbital stability of periodic solutions of autonomous systems with impulse effect, Internat. J. Sys. Sci. 19 (1988), 2562-2585.

[13] H.L. Sмith And H.R. Thieme, Dynamical systems and population persistence, Amer. Math. Soc., 2011.

[14] H.R. Thieme, Mathematics in population biology, Princeton University Press, 2003.

[15] Y. Ye, Theory of Limit Cycles, Amer. Math. Soc., Providence RI, 1986.

Changming Ding and Zhongxin Zhang

School of Mathematical Science

Xiamen University

Xiamen, Fujian 361005, P.R. CHINA

E-mail address: cding@xmu.edu.cn. 